\title{
Padrão de Prescrição de Medicamentos nas Unidades de Programa de Saúde da Família de Blumenau
}

\author{
Daniela Colombo*, Ernani Tiarajú de Santa Helena, Ana Cláudia Maciel Gava Agostinho, \\ Janaina Suzete Moreira Alcantara Didjurgeit
}

Fundação Universidade Regional de Blumenau, Santa Catarina

*Correspondência:

D. Colombo

Fundação Universidade Regional de

Blumenau

Centro de Ciências da Saúde

Rua Antônio da Veiga, 140 - Victor

Konder - Sala J-105

89010-500 - Blumenau - SC

E-mail:dncol@terra.com.br
Os medicamentos constituem parte importante dos recursos terapêuticos disponiveis e muitas vezes são a opção com melhor perfil custo-beneficio. O conhecimento do padrão de prescrição de um determinado local possibilita melhor gerenciamento da assistência farmacêutica. O objetivo do presente trabalho foi descrever os medicamentos prescritos em uma amostragem do Programa de Saúde da Família (PSF) de Blumenau, SC. Foram entrevistados 186 pacientes durante um dia de atendimento, classificando-se os dados de acordo com os indicadores de prescrição da OMS. Foram prescritos 336 medicamentos, sendo que o número médio de medicamentos por prescrição foi 1,8. A porcentagem de antibióticos foi $12,5 \%$ e de receitas com medicamentos injetáveis foi $8,1 \%$. As classes terapêticas mais prevalentes foram analgésicos $(14,3 \%)$, antibacterianos sistêmicos (12,5\%) e antiinflamatórios (12,5\%). A maioria dos medicamentos fazia parte da listagem de medicamentos padronizados pelo municipio e da RENAME $(82,4 \%$ e $57,7 \%$, respectivamente). O número de medicamentos por receita e a freqüencia de prescrição de antibióticos foram menores que aqueles encontrados em outros estudos. Além disso, a maioria dos itens prescritos fazia parte daqueles padronizados pelo município. A adoção de listagens de medicamentos essenciais e a educação continuada são ferramentas importantes para o uso racional de medicamentos.
Unitermos

- Medicamentos/prescrição

- Indicadores de prescrição

- Medicamentos essenciais

- Organização Mundial de Saúde

- Programa de Saúde da Família

\section{INTRODUÇÃO}

Medicamentos com estrutura química conhecida causaram revolução na saúde pública e no exercício da Medicina, alcançando papel central na terapêutica contemporânea (Nascimento, 2002). De acordo com Castán
Cameo et al. (1998), os custos com medicamentos representam o principal gasto no cuidado básico de saúde. Contudo, quando usados apropriadamente, os medicamentos são o recurso terapêutico mais freqüentemente custoefetivo (McIsaac et al. Apud Marin, 2003).

Considerando-se que os custos com medicamentos 
representam parte importante do cuidado à saúde e que a prescrição de medicamentos é um ato complexo, sujeito a vários erros, é fundamental, para aqueles que elaboram e implementam políticas de saúde, conhecer o padrão de prescrição vigente na área em que atuam. Esse conhecimento possibilita a adoção de estratégias de gestão e controle que tragam a melhor relação custo-benefício para a comunidade atendida (Avery, 2002; Butler, 2001; Gonzales, 2001; Latour Perez, 2000; Birrell, 2000; Millet Medina, 1998; Mainous, 1998, Marin, 2003; Mazzaglia, 1998).

De acordo com a Organização Mundial de Saúde (Hardon, Brudon-Jacobowicz, Reeler, 1992), existem vários aspectos que demonstram prescrição inadequada de medicamentos:

- Emprego inapropriado de antibióticos, em situações em que não há necessidade, como resfriados e diarréias simples;

- Utilização excessiva de fármacos injetáveis, ocasionada pela crença, tanto de profissionais de saúde quanto de pacientes, de que injeções são mais efetivas que comprimidos;

- Sobreuso de medicamentos relativamente seguros;

- Uso de combinações de fármacos, nas quais muitas vezes são associados fármacos antagônicos ou de efeito similar, sendo que o grande exemplo são os antigripais, compostos, amiúde, por dois ou mais medicamentos;

- Uso desnecessário de medicamentos de alto custo, causado pela preferência de nomes comerciais conhecidos, usualmente mais caros, em detrimento de medicamentos genéricos, usualmente mais acessíveis.

Outro elemento que afeta a prescrição e a utilização adequada dos medicamentos é a forma como estão organizadas as intervenções no processo saúde-doença. Desde o início do século 20 até os dias de hoje, o Brasil vem passando por mudanças no modelo de assistência à saúde, de acordo com as necessidades e com o entendimento vigente em cada época. Em 1988, a Constituição Federal definiu que "... a saúde é direito de todos e dever do estado...", sendo que os cuidados à saúde devem ser organizados atendendo aos princípios de universalização, integralidade, descentralização, hierarquização e participação popular. O modelo assistencial atual prioriza o atendimento básico, transformando-o em porta de entrada para todos os níveis restantes do sistema de saúde. Uma das iniciativas para o cumprimento dessas diretrizes foi a criação do Programa de Saúde da Família (PSF), em 1994. A estrutura do PSF estabelece que os habitantes de cada município sejam divididos em grupos com área geográfica e população definidas. Cada grupo deve conter aproxima- damente 4.500 pessoas, que serão atendidas por uma equipe de saúde composta por, no mínimo, um médico, um enfermeiro, dois auxiliares de enfermagem e 4 a 5 agentes de saúde. Conforme o Ministério da Saúde (Brasil, 2001), “... o PSF tem condições de dar solução efetiva a mais de $85 \%$ dos problemas de saúde da população atendida".

Em Blumenau, SC, a primeira equipe de PSF iniciou seu funcionamento em 1995 e conta até hoje com o mesmo profissional médico. Desde lá, foram implantadas mais 30 equipes, sendo que, atualmente, $46 \%$ da população blumenauense é atendida pelo PSF.

Ao longo dos anos, com a identificação de medicamentos como uma modalidade terapêutica fundamental, vários esforços têm sido realizados para promover seu uso racional, diminuindo os custos associados com prescrições inadequadas, efeitos adversos e utilização incorreta. Um conceito introduzido pela OMS nos anos 1970 contempla a adoção de listagens de medicamentos essenciais de acordo com as necessidades locais (Marin, 2003). Com o objetivo de racionalizar o uso dos medicamentos existentes, o Ministério da Saúde criou uma lista de medicamentos denominada Relação Nacional de Medicamentos Essenciais (RENAME) (Brasil, 2002), que visa orientar o uso de produtos seguros, eficazes e com possibilidade de solucionar a maior parte dos problemas de saúde da população brasileira. Baseado na RENAME e levando em conta as particularidades municipais, a cidade de Blumenau criou a Relação Municipal de Medicamentos Essenciais (REMUME). Todos os medicamentos da REMUME são fornecidos gratuitamente, mediante prescrição médica, em todas as unidades de saúde de Blumenau.

Essa pesquisa objetiva descrever quais os medicamentos prescritos no PSF de Blumenau, a quantidade de medicamentos por receita, a percentagem de utilização de antibióticos e fármacos injetáveis e a utilização de fármacos da REMUME e RENAME.

\section{MATERIAL E MÉTODOS}

\section{Amostragem}

Foi realizada uma pesquisa nas equipes de PSF do município de Blumenau, sendo sorteado o dia 12 de novembro de 2003 para a coleta de dados. Nesse dia, foram entrevistados todos os pacientes que passaram por consulta médica e receberam prescrição de medicamento. Todas as 31 equipes existentes em Blumenau naquela data foram arroladas para a pesquisa. Entretanto, em catorze equipes houve impossibilidade da coleta dos dados por razões diversas: ausência do médico no dia da pesquisa (2 equipes); 
não concordância da equipe com a pesquisa (6 equipes) e ausência de uma das auxiliares de enfermagem da equipe (6 equipes).

\section{Tamanho da Amostra}

Para o cálculo da amostra utilizou-se a fórmula descrita por Luiz e Magnanini (2003), que levou em conta os seguintes parâmetros:

- População cadastrada no PSF do município;

- Prevalência observada na literatura para as principais variáveis em estudo (porcentagem de receitas com antibióticos e injeções, além da freqüência de utilização dos medicamentos da RENAME) e

- Intervalo de confiança de 95\%.

Dessa forma, o tamanho da amostra foi calculado em 322 sujeitos. Entretanto, pelas razões acima descritas, foi atingida a amostragem de 186 pessoas.

\section{Coleta dos Dados}

Os dados foram coletados por um dos auxiliares de enfermagem da equipe de PSF na qual o paciente foi atendido, imediatamente após a consulta médica. O entrevistador foi sorteado e treinado previamente. Nenhum paciente negou-se a responder. A pesquisa foi aprovada pelo Comitê de Ética na Pesquisa em Seres Humanos da Universidade Regional de Blumenau e pela Secretaria de Saúde do município de Blumenau.

No formulário de entrevista constavam as seguintes perguntas, objetivando a caracterização da amostragem e comparação com outros estudos: sexo, idade e motivo da consulta. A seguir, todos os medicamentos prescritos para aquele paciente foram transcritos para o mesmo formulário, da maneira como foram registrados nas receitas médicas.

\section{Inclusão e Exclusão}

Todas as receitas prescritas na consulta imediatamente anterior à entrevista foram utilizadas no estudo, inclusive aquelas que continham medicamentos não inclusos na REMUME.

Não foram computadas as prescrições para medicamentos de uso contínuo (como anti-hipertensivos, por exemplo) não emitidas no dia da consulta.

\section{Análise dos Dados}

Para fins de análise dos dados, considerou-se que todos os medicamentos prescritos para um determinado paciente faziam parte da mesma receita.
Os dados foram analisados utilizando-se o software Epi Info 2002, dos Centros para Prevenção e Controle de Doenças (Centers for Disease Control and Prevention), nos Estados Unidos. A análise dos dados foi feita de forma descritiva, mediante a elaboração de tabelas.

Os motivos de consulta foram descritos de acordo com a segunda edição da Classificação Internacional de Atenção Primária (International Classification of Primary Care - ICPC-2), desenvolvida pela Organização Mundial de Médicos de Família (World Organization of National Colleges, Academies and Academic Associations of General Practitioners/Family Physicians - WONCA) (WONCA, 1998). Embora a Classificação Internacional de Doenças (CID) seja mundialmente aceita para uniformizar os diagnósticos possíveis, ela ainda sofre com os vieses de sua origem hospitalar. Dessa forma, não é um instrumento muito útil em atenção primária, uma vez que se trata de compêndio de todos os diagnósticos existentes, muito deles incomuns e raramente, ou nunca, vistos no atendimento básico. A ICPC-2 é compatível com a CID e mais prática para a codificação de diagnósticos em atenção primária (Starfield, 2002).

Os medicamentos foram apresentados pela nomenclatura constante na Denominação Comum Brasileira (ANVISA, 2003). Foram, ainda, classificados pelo Índice de Classificação Anatômico, Químico e Terapêutico (Anatomical-Therapeutical-Chemical Classification Index - ATC) (WHO, 2004), pela RENAME, do Ministério da Saúde (Brasil, 2002) e pela REMUME, da Secretaria de Saúde de Blumenau (Blumenau, 2003).

Utilizaram-se, ainda, os indicadores de prescrição recomendados pelo Centro Colaborador de Métodos Estatísticos em Medicamentos da Organização Mundial da Saúde (Hardon, Brudon-Jacobowicz, Reeler, 1992), a saber:

- Número médio de medicamentos por receita;

- Porcentagem de consultas em que se prescrevem antibióticos;

- Porcentagem de medicamentos injetáveis prescritos.

Também, foram utilizados os indicadores que seguem, selecionados de estudos similares (Diez Córdova et al., 1997; Fegadolli, Mendes, Simões, 2002; Nascimento Jr, 1999);

- Porcentagem de medicamentos prescritos que constam na RENAME e REMUME;

- Porcentagem de receitas com psicotrópicos.

\section{RESULTADOS}

Entrevistaram-se 186 usuários de 17 equipes de PSF de Blumenau, sendo a maioria $(66,1 \%)$ constituída pelo 
sexo feminino. A média de idade das mulheres foi 30,4 anos, significativamente maior ( $\mathrm{p} 0,01)$ que a média dos homens, que foi de 22,1 anos.

Quanto à idade, a faixa etária mais freqüente foi a de 20 a 39 anos, com 32,8\% do total de atendimentos (Tabela I). Mais da metade dos pacientes atendidos $(54,3 \%)$ tinha entre 20 e 59 anos.

Classificando-se os motivos para consulta, conforme a Classificação Internacional para Atenção Primária, as principais causas foram relacionadas ao sistema respira- tório $(14,1 \%)$ e músculo-esquelético $(13,5 \%)$. Os dados estão descritos na Tabela II.

Os medicamentos mais prescritos foram diclofenaco e paracetamol, respondendo ambos por $22,6 \%$ do total. Outros medicamentos prescritos estão discriminados na Tabela III.

Diclofenaco foi o medicamento mais prescrito para ambos os sexos $(9,6 \%$ dos 229 medicamentos receitados para o sexo feminino e $15 \%$ dos 107 medicamentos receitados para o sexo masculino).

TABELA I - Idade média da população atendida de acordo com as faixas etárias do SIAB

\begin{tabular}{lccc}
\hline Faixa Etária & TotalN & Freqüência Relativa\% & Intervalo de Confiança (95\%)\% \\
\hline Menor de 1 ano & 13 & 7,0 & $3,8-11,7$ \\
1 a 4 anos & 17 & 9,1 & $5,4-14,2$ \\
5 a 9 anos & 18 & 9,7 & $5,8-14,9$ \\
10 a 14 anos & 10 & 5,4 & $2,6-9,7$ \\
15 a 19 anos & 12 & 6,4 & $3,4-11,0$ \\
20 a 39 anos & 61 & 32,8 & $26,1-40,0$ \\
40 a 49 anos & 25 & 13,4 & $8,9-19,2$ \\
50 a 59 anos & 15 & 8,1 & $4,6-13$ \\
Maior de 60 anos & 15 & 8,1 & $4,6-13$ \\
\hline Total & 186 & 100,0 & \\
\hline
\end{tabular}

Fonte: Dados primários, PSF Blumenau - 2003.

TABELA II - Motivos para consulta mais freqüentes da população atendida segundo grupos $I C P C-2$

\begin{tabular}{|c|c|c|c|c|c|}
\hline $\begin{array}{l}\text { Grupo } \\
\text { ICPC-2 }\end{array}$ & Motivos Agrupados & $\begin{array}{l}\text { Total } \\
\mathbf{N}\end{array}$ & $\begin{array}{c}\text { Freqüência } \\
\text { Relativa } \\
\%\end{array}$ & $\begin{array}{c}\text { Freqüência } \\
\text { Cumulativa } \\
\%\end{array}$ & $\begin{array}{c}\text { Intervalo de } \\
\text { Confiança } \\
(95 \%) \%\end{array}$ \\
\hline $\mathrm{R}$ & Respiratório & 42 & 14,1 & 14,1 & $10,4-18,6$ \\
\hline $\mathrm{L}$ & Músculo-esquelético & 40 & 13,5 & 27,6 & $9,8-17,9$ \\
\hline A & Geral e inespecífico & 38 & 12,8 & 52,9 & $9,2-17,1$ \\
\hline$*$ & Procedimentos & 37 & 12,5 & 40,1 & $8,9-16,8$ \\
\hline $\mathrm{D}$ & Digestivo & 34 & 11,4 & 64,3 & $8,1-15,6$ \\
\hline $\mathrm{N}$ & Neurológico & 19 & 6,4 & 70,7 & $3,9-9,8$ \\
\hline S & Pele & 15 & 5,1 & 75,8 & $2,9-8,2$ \\
\hline $\mathrm{T}$ & Endócrino/metabólico/ nutricional & 10 & 3,4 & 79,2 & $1,6-6,1$ \\
\hline $\mathrm{U}$ & Urológico & 10 & 3,4 & 82,6 & $1,6-6,1$ \\
\hline $\mathrm{W}$ & Gestação/parto/planejamento familiar & 10 & 3,4 & 86,0 & $1,6-6,1$ \\
\hline $\mathrm{H}$ & Otológico & 9 & 3,0 & 89,0 & $1,4-4,7$ \\
\hline $\mathrm{X}$ & Genital feminino & 9 & 3,0 & 92,0 & $1,4-5,7$ \\
\hline $\mathrm{P}$ & Psicológico & 8 & 2,7 & 94,7 & $1,2-5,2$ \\
\hline K & Cardiovascular & 7 & 2,4 & 97,1 & $1,0-4,8$ \\
\hline B & Sangue & 6 & 2,0 & 99,0 & $0,7-4,3$ \\
\hline Y & Genital masculino & 1 & 0,3 & 99,4 & $0,0-1,9$ \\
\hline Total & & 297 & 100,0 & 100,0 & \\
\hline
\end{tabular}

Fonte: Dados primários, PSF Blumenau - 2003. 
Em conformidade com os medicamentos mais prescritos, as classes terapêuticas mais prevalentes foram analgésicos $(14,3 \%)$, antibacterianos sistêmicos $(12,5 \%)$ e antiinflamatórios (12,5\%). As classes mais prescritas encontram-se na Tabela IV.

A via de administração mais utilizada foi a oral (268), correspondendo a $79,8 \%$ do total, conforme descrito na Tabela V.
Os indicadores de prescrição estão na Tabela VI. Observou-se que todas as prescrições de medicamentos psicotrópicos foram para pacientes do sexo feminino.

\section{DISCUSSÃO}

A coleta de dados foi realizada em cinco das seis regiões administrativas da Secretaria de Saúde do municí-

TABELA III - Medicamentos mais prescritos na amostragem avaliada

\begin{tabular}{lcccc}
\hline Medicamento & Total & $\begin{array}{c}\text { Freqüência } \\
\text { Relativa } \\
\text { \% }\end{array}$ & $\begin{array}{c}\text { Freqüência } \\
\text { Cumulativa } \\
\text { \%o }\end{array}$ & $\begin{array}{c}\text { Intervalo de } \\
\text { Confiança (95\%) } \\
\text { \% }\end{array}$ \\
\hline Diclofenaco & 38 & 11,3 & 11,3 & $8,2-15,3$ \\
Paracetamol & 38 & 11,3 & 22,6 & $8,2-15,3$ \\
Dexclorfeniramina & 14 & 4,2 & 26,8 & $2,4-7,1$ \\
Amoxicilina & 11 & 3,3 & 30,1 & $1,7-6,6$ \\
Metoclopramida & 10 & 3,0 & 33,1 & $1,5-5,6$ \\
Dipirona & 9 & 2,7 & 35,8 & $1,3-5,2$ \\
Miconazol & 9 & 2,7 & 38,5 & $1,3-5,2$ \\
Salbutamol & 9 & 2,7 & 41,2 & $1,3-5,2$ \\
Sulfato ferroso & 9 & 2,7 & 43,9 & $1,3-5,2$ \\
Fluconazol & 8 & 2,4 & 46,3 & $1,1-4,8$ \\
Metronidazol & 8 & 2,4 & 48,7 & $1,1-4,8$ \\
Cefalexina & 7 & 2,1 & 50,8 & $0,9-4,4$ \\
Soro fisiológico nasal com & 7 & 2,1 & 52,9 & $0,9-4,4$ \\
$\quad$ cloreto de benzalcônio & & 47,3 & 100,0 & $41,9-52,8$ \\
Outros & 159 & 100,0 & 100,0 & \\
\hline Total & 336 & &
\end{tabular}

Fonte: Dados primários, PSF Blumenau - 2003.

TABELA IV - Classes terapêuticas mais prescritas na amostragem avaliada

\begin{tabular}{|c|c|c|c|c|c|}
\hline $\begin{array}{l}\text { Classe } \\
\text { ATC }\end{array}$ & Classes Terapêuticas & $\begin{array}{l}\text { Total } \\
\mathbf{N}\end{array}$ & $\begin{array}{c}\text { Freqüência } \\
\text { Relativa } \\
\%\end{array}$ & $\begin{array}{c}\text { Freqüência } \\
\text { Cumulativa } \\
\%\end{array}$ & $\begin{array}{c}\text { Intervalo de } \\
\text { Confiança } \\
(95 \%) \%\end{array}$ \\
\hline N02 & Analgésicos & 48 & 14,3 & 14,3 & $10,8-18,6$ \\
\hline J01 & Antibacterianos sistêmicos & 42 & 12,5 & 26,8 & $9,3-16,6$ \\
\hline M01 & Antiinflamatórios & 42 & 12,5 & 39,3 & $9,3-16,6$ \\
\hline A03 & $\begin{array}{l}\text { Drogas para distúrbios funcionais do } \\
\text { aparelho digestivo }\end{array}$ & 24 & 7,1 & 46,4 & $4,7-10,6$ \\
\hline R03 & Broncodilatadores e antiasmáticos & 17 & 5,1 & 51,5 & $3,1-8,1$ \\
\hline R06 & Antitussígenos e antigripais & 15 & 4,5 & 56,0 & $2,6-7,4$ \\
\hline B03 & Antianêmicos & 9 & 2,7 & 62,0 & $1,3-5,2$ \\
\hline Outros & & 128 & 38,1 & 100,0 & $32,9-43,5$ \\
\hline Total & & 336 & 100,0 & 100,0 & \\
\hline
\end{tabular}

Fonte: Dados primários, PSF Blumenau - 2003. 
TABELA V - Freqüência de utilização das diferentes vias de administração na amostragem avaliada

\begin{tabular}{lcccc}
\hline Via & Total & $\begin{array}{c}\text { Freqüência } \\
\text { Relativa } \\
\mathbf{\%}\end{array}$ & $\begin{array}{c}\text { Freqüência } \\
\text { Cumulativa } \\
\mathbf{\%}\end{array}$ & $\begin{array}{c}\text { Intervalo de } \\
\text { Confiança (95\%) } \\
\mathbf{\%}\end{array}$ \\
\hline Oral & $\mathbf{N}$ & 79,8 & 79,8 & $75,1-83,9$ \\
Tópica & 268 & 11,6 & 91,4 & $8,5-15,6$ \\
Intramuscular & 39 & 3,6 & 95,0 & $1,9-6,3$ \\
Vaginal & 12 & 2,4 & 97,4 & $1,1-4,8$ \\
Inalatória & 8 & 1,8 & 99,2 & $0,7-4,0$ \\
Intravenosa & 6 & 0,9 & 100,0 & $0,2-2,8$ \\
\hline Total & 3 & 100,0 & 100,0 & \\
\hline
\end{tabular}

Fonte: Dados primários, PSF Blumenau - 2003.

TABELA VI - Indicadores de prescrição utilizados na pesquisa

\begin{tabular}{|c|c|c|}
\hline Indicadores & $\begin{array}{c}\text { Total } \\
n^{1}(\mathbf{N})^{2} \\
\end{array}$ & $\begin{array}{c}\text { Freqüência Relativa } \\
\%\end{array}$ \\
\hline Número de prescrições com antibacterianos sistêmicos & $42(336)$ & 12,5 \\
\hline Número de prescrições com injetáveis & $15(336)$ & 8,1 \\
\hline Número de prescrições com psicotrópicos & $6(336)$ & 3,2 \\
\hline Numero de medicamentos que pertencem a RENAME & $194(336)$ & 57,7 \\
\hline Numero de medicamentos que pertencem a REMUME & $277(336)$ & 82,4 \\
\hline
\end{tabular}

\section{Número médio de medicamentos por receita: 1,8}

Fonte: Dados primários, PSF Blumenau - 2003.

1. Refere-se ao total do indicador em questão; 2 . Refere-se ao total de medicamentos prescritos.

pio, constituindo distribuição abrangente das equipes. Uma vez que cada equipe de PSF tem autonomia para organizar sua agenda diária, a escolha aleatória de um dia para a coleta de dados possibilitou entrevistar indivíduos oriundos de diferentes faixas etárias, sexo e modalidades de atendimento (demanda espontânea, agendamentos e grupos). Tal fato sugere que a amostragem representa a população atendida em um dia nos PSFs de Blumenau.

$\mathrm{Na}$ amostra pesquisada, foram relatados 297 motivos para consulta. A grande variedade de motivos para a consulta é compatível com o atendimento primário, no qual, segundo Starfield (2002), deve haver série ampla de problemas apresentados pelos usuários, em contraste com o atendimento especializado, em que pequena variedade de diagnósticos corresponde à maioria das consultas.

O grupo de motivos mais freqüente, segundo a classificação da WONCA, foi o de queixas respiratórias $(14,1 \%)$, seguido pelo de queixas músculo-esqueléticas (13,5\%). Também Medina Lois et al. (1998), num estudo realizado sobre atenção primária, em Santiago do Chile, mostram um predo- mínio de consultas respiratórias e do aparelho locomotor. É difícil comparar esses dados com outros estudos, visto que a maioria enfoca o diagnóstico e não a queixa.

Não é possível estabelecer a correção da prescrição em relação à patologia apresentada pelo paciente, uma vez que para tal seria necessário revisar o prontuário do paciente ou entrevistar o próprio médico, a fim de estabelecer o diagnóstico preciso, o que não foi proposto nesse estudo.

No total, foram prescritos 336 medicamentos. Os mais receitados no município de Blumenau, nessa amostragem, foram diclofenaco e paracetamol, com 38 prescrições cada um (11,3\% cada). As classes terapêuticas mais prescritas foram, em concordância com os medicamentos mais prescritos, analgésicos (14,3\%) e antiinflamatórios (12,5\%). Em outros estudos, as classes terapêuticas mais prescritas foram aquelas que correspondem a fármacos com ação no sistema respiratório (Carneiro, Marques, Simões, 2000; Fegadolli, Mendes, Simões, 2002). Essa discrepância talvez se deva ao fato de esses estudos avaliarem apenas prescrições pediátricas. 
Já em uma avaliação de 2400 indivíduos que consultaram com médicos de família, incluindo adultos e crianças, realizada em Cuba (Diez Córdova et al., 1997), 34,6\% dos medicamentos prescritos eram analgésicos.

Nas prescrições avaliadas, encontraram-se 6 receitas que continham antidepressivos ou ansiolíticos, o que corresponde a 3,2\% do total de 186 receitas. Apesar de encontrarmos poucas receitas com psicotrópicos, é interessante notar que $100 \%$ delas foram para o sexo feminino. Isso pode ser explicado pela maior freqüência de consultas de mulheres nesta amostra. Além disso, esses dados podem sugerir maior prevalência de transtornos mentais no sexo feminino ou indicar que as mulheres são mais propensas que os homens a procurar atendimento por queixas relacionadas a transtornos mentais.

Dos 336 medicamentos prescritos, a maioria absoluta $(82,4 \%)$ fazia parte da REMUME. Em Araraquara, SP, $61,35 \%$ dos medicamentos constavam da listagem padronizada do município (Simões, Falvo, 2000). Em Florianópolis, em 1999, Nascimento Jr. verificou que 86,5\% dos medicamentos prescritos constavam da lista municipal de medicamentos padronizados. Esses achados contrastam com aquele apresentado por Fegadolli, Mendes e Simões (2002), que relatam porcentagem de medicamentos prescritos que constavam na lista de medicamentos do município de Tabatinga, SP, de apenas $22,8 \%$. Isso pode significar que os médicos dos PSFs de Blumenau procuram receitar os medicamentos fornecidos pelo município. Alem disso, também sugere que a lista de medicamentos padronizados do município de Blumenau atende a maioria das necessidades da população. Quanto à importância dos medicamentos, pouco mais da metade $(57,7 \%)$ dos prescritos fazia parte da RENAME. Isso pode ser explicado pelo fato de um dos medicamentos mais prescritos (diclofenaco) não fazer parte desta lista (os antiinflamatórios não-esteróides recomendados pela RENAME são o ácido acetilsalicílico e o ibuprofeno).

$\mathrm{Na}$ amostragem pesquisada, a média de medicamentos por prescrição foi de 1,8 , similar à média descrita por Costa, Macedo e Hermógenes (1992), que foi de 1,7 medicamentos por prescrição, e inferior às médias descritas por Santos (1999), Simões e Falvo (2000) e Carneiro, Marques e Simões (2000), que foram de 2,4, 2,6 e 2,9 medicamentos por prescrição, respectivamente.

Das 186 prescrições avaliadas, 42 continham antibióticos, contabilizando 22,6\%. Comparando com dados publicados no Brasil e restante da América Latina, somente Simões e Falvo (2000) descrevem taxa nitidamente inferior de prescrição de antibióticos $(5,4 \%)$, possivelmente porque o estudo avalia as prescrições de idosos durante um período de 4 meses, para os quais aparecem com maior freqüência medicamentos para doenças crônicodegenerativas. Santos (1999) encontrou taxa semelhante à Blumenau (21,3\%) e Carneiro, Marques e Simões (2000) encontraram 33,9\% de receitas com antibióticos no município de Américo Brasiliense, em São Paulo. No município de Tabatinga, SP, Fegadolli, Mendes e Simões (2002) relataram $44,6 \%$ de receitas com antibióticos. Em uma pesquisa feita na Bolívia (Pinard, Bacherer, 1996), metade das receitas continha um antibiótico.

O número de receitas que continham medicamentos injetáveis foi de 15 (8,1\%). Também, aqui, Simões e Falvo (2000) descrevem taxa bastante inferior: $4,2 \%$, pelos mesmos motivos descritos anteriormente. Outros pesquisadores relatam taxas similares a Blumenau: 9,9\% (Carneiro, Marques e Simões, 2000) e 10,4\% (Fegadolli, Mendes e Simões, 2002). Já Pinard e Bacherer (1996) relataram que um terço das receitas prescritas em Santa Cruz, na Bolívia, continham medicamentos injetáveis.

Não existe um padrão de prescrição fixo, uma vez que esse depende de características do sistema de saúde e da população avaliada, podendo variar sobremaneira, mesmo de município para município. Contudo, conforme apresentado anteriormente, em Blumenau prescrevem-se menos medicamentos por receita e utilizam-se menores quantidades de fármacos injetáveis e de antibióticos. Também são utilizados prioritariamente os medicamentos da REMUME. Esses índices podem refletir a preocupação da Secretaria de Saúde em manter um programa regular de educação continuada, compulsório, que inclui todos os profissionais do PSF. Neste programa regular são abordados diversos assuntos, entre eles as patologias mais prevalentes e seu tratamento. Outro dado que talvez explique a melhor posição com respeito aos índices apresentados em outros estudos pode ser a relação próxima entre a rede municipal de saúde e a Faculdade de Medicina local, pois muitos profissionais fazem parte das duas instituições.

É interessante ressaltar que os dados aqui apresentados referem-se a novas prescrições ou repetição de prescrições anteriores para pacientes que consultaram no dia da pesquisa. Em Blumenau, existe a chamada "receita de uso contínuo", que inclui anti-hipertensivos e antidiabéticos, p.e. Nesse caso, é fornecida uma receita, com validade de seis meses, para pacientes com controle adequado de doenças crônicas e que não necessitem de reavaliação mais freqüente. Durante esses seis meses, o controle do fornecimento dos medicamentos é realizado pelas auxiliares de enfermagem de cada equipe, não sendo necessária a emissão de receita médica. Dessa forma, a baixa freqüência de anti-hipertensivos apresentada nessa pesquisa provavelmente não reflete a prevalência do uso desse tipo de medicamento no município. 


\section{CONCLUSÃO}

O estudo realizado mostra que, em relação à maior parte dos indicadores, o PSF do município de Blumenau se encontra em situação melhor que a maioria das outras localidades pesquisadas, ou seja, prescrevem-se menos medicamentos por receita, utilizam-se menores quantidades de fármacos injetáveis e de antibióticos. Além disso, a maioria dos itens prescritos fazia parte daqueles padronizados pelo município. A utilização de medicamentos padronizados melhora a relação custo-benefício da prescrição, uma vez que se utilizam fármacos de eficácia e segurança estabelecidas, adquiridos através de concorrência pública, pelo menor preço possível.

A adoção de uma listagem padronizada de medicamentos e a existência de um programa institucional de educação continuada são instrumentos que promovem o uso racional de medicamentos e devem ser fazer parte do gerenciamento de saúde dos municípios.

A prescrição de medicamentos e os problemas relacionados a ela são assuntos de grande complexidade. A utilização de indicadores padronizados para a pesquisa, como aqueles sugeridos pela OMS, facilita a coleta de dados de maneira organizada e passível de comparação. Infelizmente, ainda são poucos os estudos no Brasil que utilizam esses indicadores.

\section{ABSTRACT}

\section{Prescribed Drugs in Attendance Sampling at Basic Health Service in Blumenau}

Medicines constitute important part of available therapeutic resources and are often the option with better cost/benefit profile. The knowledge of the prescription pattern from a determined local allows better management of pharmaceutical assistance. The objective of this work was to list prescribed drugs in an attendance sample in the city of Blumenau, Santa Catarina. With this purpose 186 patients were interviewed in 17 health centers during a working day, using data classification according to WHO prescribing indicators. In this study 336 drugs were prescribed and the drug average per prescription was 1,8. Antibiotic prescription percentage was 12,5 and the percentage was 8,1 for injections. The most prevalent therapeutic subgroups were analgesics (14,3\%), systemic antibiotics $(12,5 \%)$ and anti-inflammatory (12,5\%). The majority of these drugs was part of the municipal standardized medication list and national list of essential drugs RENAME (82,4\% and 57,7\%, respectively). The amount of drugs per prescription and antibiotics prescription frequency were lower then those found in other researches. Besides, most of the prescribed drugs were part of those standards from the city. The adoption of essential drug lists and a continuing education are important tools for the rational use of medicines.

UNITERMS: Medicines/prescription. Prescription indicators. Essential drugs. World Health Organization; Family Health Program.

\section{REFERÊNCIAS BIBLIOGRÁFICAS}

ANVISA. Medicamentos. Conceitos técnicos. Brasília, 2000. Disponível em: $<$ http://www.anvisa.gov.br/ medicamentos/conceito.htm>. Acesso em: 8 abr. 2003.

AVERY, A. J. et al. Safer medicines management in primary care. Br. j. gen. practice, v. 52, supl.1, n. 484, p. 17-21, 2002.

BIRRELL, G.; BIRRELL, R. G. Assessment of a 1-year teaching programme in Zanzibar, Tanzania. Lancet, v. 356, n. 9235 , p. 1084, 2000.

BLUMENAU. Prefeitura Municipal de Blumenau. Secretaria Municipal de Saúde. Relação municipal de medicamentos essenciais. Blumenau, 2003. (Mimeo).

BRASIL. Constituição da República Federativa do Brasil. 27. ed. São Paulo: Saraiva, 2001. 331 p.

BRASIL. Ministério da Saúde. Agência Nacional de Vigilância Sanitária. Portaria n. 1.179, de 17 de junho de 1996. Denominações Comuns Brasileiras DCB. Diário Oficial [da] República Federativa do Brasil. Brasília, DF, 18 jun. 1996. Disponível em: < http:// www.anvisa.gov.br/legis/portarias/1179 96.htm>. Acesso em: 8 abr 2003.

BRASIL. Ministério da Saúde. Departamento de Atenção Básica. Guia prático do programa de saúde da família. Brasília, Ministério da Saúde, 2001. 128 p.

BRASIL. Ministério da Saúde. Gabinete do Ministro. Portaria n. 1.587 , de 3 de setembro de 2002. Relação Nacional de Medicamentos Essenciais RENAME - Revisão. Diário Oficial [da] República Federativa do Brasil. Brasília, DF, 5 setembro de 2002. Disponível em: <http:// www.anvisa.gov.br/legis/portarias/1587 02.htm>. Acesso em: 29 fev. 2004. 
BRASIL. Ministério da Saúde. Sistema de Informações da Atenção Básica. Cadastramento familiar em Blumenau, Santa Catarina, em agosto de 2004. Disponível em: $<$ http:// www.datasus.gov.br/siab/siab.htm>. Acesso em: 21 de nov. de 2004.

BUTLER, C. C. et al.Antibiotics and shared decision making in primary care. J. Antimicrob. Chemother., v. 48, n. 3, p. 435-440, 2001.

CARNEIRO, R. M.; MARQUES, M. C. P.; SIMÕES, M. J. S. Estudo das prescrições de medicamentos em crianças de 0 a 2 anos atendidas no serviço municipal de saúde de Américo Brasiliense - SP, 1999. Rev. Ciênc. Farm., v. 21, n. 2, p. 229-248, 2000.

CASTÁN CAMEO, S.; GARCIA LATORRE, F. J.; MARTINEZ GOROSTIAGA, J.; SIERRA MOROS, M. J.; SOLANO BERNAD, U. M.; PERAL CASADO, A. Un estudio de minimización de costes en la prescripción de antiinfecciosos en dos áreas de atención primaria. Rev. Esp. Salud Publica, v. 72, n. 1, p. 33-42, 1998.

COSTA, M. da C. N.; MACEDO, J. N.; HERMÓGENES, J.A. Prescrição, dispensação e uso de medicamentos em dois centros de saúde de Salvador. Rev. Baiana Saúde Pública, v. 19, n. 1, p. 19-24, 1992

DIEZ CÓRDOVA, J. P.; DIEZ CÓRDOVA, J. P.; DEBESA GARCIA, F.; SOSA LORENZO, I. A; GARCIA ROCHE, R. G.; BONET GORBEA, M. H.; FERNÁNDEZ LARREA, N. Estado de la precripción de medicamentos en la atención primaria de salud de ciudad de la habana, 1997. Rev. cubana med. gen. integr. Habana, v. 15, n.2, p. 140-150. Disponível em: $<$ http://scielo.sld.cu/scielo.php ? s c ript $=\mathrm{sci}$ arttext \& pid $=\mathrm{S} 0864-$ $21251999000200006 \& \operatorname{lng}=\mathrm{en} \& \mathrm{nrm}=\mathrm{iso}>$. Acesso em: 29 fev. 2004.

FEGADOLLI, C.; MENDES, I. J. M.; SIMÕES, M. J. S. Avaliação da prescrição médica em pediatria, baseada nos indicadores de medicamentos selecionados pela OMS em município do interior do estado de São Paulo. Rev. Ciênc. Farm., v. 23, n. 2, p. 239-254, 2002.

GONZALES, R.; MANOLE, D. C.; MASELLI, J. H.; SANDE, M. A. Excessive antibiotic use for acute respiratory infections in the United States. Clin. Infect. Dis., v. 33, n. 6, p. 757-762, 152001.
HARDON, A.; BRUDON-JAKOBOWICZ, P.; REELER, A. How to investigate drug use in communities. Guidelines for social science research. World Health Organization, Geneva, 1992. Disponível em: $<$ http:// whqlibdoc.who.int/hq/1992/WHO_DAP_92.3.pdf>. Acesso em: 23 abr. 2003.

KOROLKOVAS, A.; FRANÇA, F. F. A. C. Dicionário terapêtico Guanabara. 7. ed. Rio de Janeiro: Guanabara Koogan, 2000.

LATOUR PEREZ, J.; DÍAZ MONDÉJAR, R.;VEGA ORTEGA, A. I.; CARRIÓN CARRIÓN, C.; GARCÍA HERRERA, J. L.; PEÑARROJA OTERO, C.; CABADÉS O'CALLAGAN, A.; PÉREZ-HOYOS, S. Fracaso de una intervención educativa para modificar la prescripción de hipocolesterolemiantes en atención primaria. Aten. Primaria, v. 26, n. 4, p. 245-8, 2000.

LUIZ, R. R.; MAGNANINI, M. M. F. O tamanho da amostra em investigações epidemiológicas. In MEDRONHO, R. A., ed. Epidemiologia. São Paulo: Editora Atheneu, 2002. 493 p.

MAINOUS, A. G.; HUESTON, W. J.; LOVE, M. M. Antibiotics for colds in children: who are the high prescribers? Arch. Pediatr. Adolescent Med., v. 152, n. 4,p. 349-352, 1998.

MARIN, N.; LUIZA, V. L.; OSORIO-DE-CASTRO, C. G. S.; MACHADO-DOS-SANTOS, S. Assistência farmacêutica para gerentes municipais. Brasília: OPAS, 2003. Disponível em: <http://www.opas.org.br/ medicamentos/>. Acesso em: 13 nov. 2004.

MAZZAGLIA, G.; GRECO, S.; LANDO, C.; CUCINOTTA, G.; CAPUTI, A. P. Adult acute upper respiratory tract infections in Sicily: pattern of antibiotic drug prescription in primary care. J. antimicrob. chemother., v. 41, n. 2, p. 259266, 1998.

MEDINA LOIS, E.; KAEMPFFER RAMÍREZ, A. M.; CORNEJO ARIAS, E.; HERNÁNDEZ, A. E. Atención primaria:evaluación de costo-efectividad. Cuad. Méd. Soc., vol. 39, n.1, p.74-79, 1998.

MILLET MEDINA, F. J.; GRACIA AGUIRRE, S.; MADRIDEJOS MORA, R.; SOLÉ LÓPEZ, J. Consumo de antibióticos (1993-1996) en la atención primaria de un área sanitaria con una tasa elevada de resistencias bacterianas. Aten. Primaria, v. 21, n. 7, p. 451-7, 1998. 
NASCIMENTO JUNIOR, J. M. Avaliação da Assistência Farmacêutica na Rede Pública Municipal de Florianópolis. Florianópolis, 1999. 200 p. [Tese de Mestrado. Centro de Ciências da Saúde. Universidade Federal de Santa Catarina].

NASCIMENTO, M. C. A centralidade do medicamento na terapêutica contemporânea. Rio de Janeiro, 2002. 139 p. [Tese Doutorado em Saúde Pública. Instituto de Medicina Social, Universidade do Estado do Rio de Janeiro].

NIES, A. S. Principles of therapeutics. In: HARDMAN, J. G.; LIMBIRD, L. E.; GILMAN, A. G., eds. Goodman and Gilman's the pharmacological basis of therapeutics. 10 ed. New York: McGraw-Hill, 2001. cap. 3, p. 45-66.

SANTOS, V. Indicadores selecionados do uso de medicamentos - OMS, no município de Ribeirão Preto-SP. São Paulo, 1999. 125p. [Tese de Doutorado em Saúde Pública. Faculdade de Saúde Pública. Universidade de São Paulo].
SIMÕES, M. J. S; FALVO, I. F. Estudo da prescrição de medicamentos para idosos atendidos em serviço público de saúde, em município da região sudeste. Rev. Ciênc. Farm., v. 21, n. 2, p. 217-227, 2000.

STARFIELD, B. Atenção primária: equilíbrio entre necessidades de saúde, serviços e tecnologia. Brasília: UNESCO, 2002. 725 p.

WHO. Collaborating Centre for Drug Statistics Methodology. ATC Index 2004. Disponível em: <http://www.whocc.no/ atcddd > Acesso em: 29 fev. 2004.

WONCA. Wonca International Classification Committee. International Classification of Primary Care Second Edition. 1998. Disponível em: <www.fmrc.org.au/ Download/ICPC-2\%20summary\%20-\%20English.pdf $>$. Acesso em: 28 fev. 2004.

Recebido para publicação em 30 de janeiro de 2004. Aceito para publicação em 28 de outubro de 2004. 